Abbreviations:

$\mathrm{PL}=$ Plenary; $\mathrm{LO}=$ Lightning oral; GD = Grizzly den;

$\mathrm{MP}=$ Moderated poster; $\mathrm{P}=$ Poster

*Corresponding authors are underlined.

\section{Plenary Oral Presentations}

\section{PL01}

Creation of the Canadian Heart Failure Risk Scale for acute heart failure patients

I.G. Stiell, MD, MSc, C.M. Clement, J.J. Perry, MD, MSc, R.J. Brison, MD, A. McRae, MD, B.H. Rowe, MD, MSc, B. Borgundvaag, MD, S. Aaron, MD, L. Mielneczuk, MD, L. Calder, MD, MSc, J. Brinkhurst, BSc, A. Forster, MD, G.A. Wells, PhD, University of Ottawa, Ottawa, ON

Introduction: Acute heart failure (AHF) is a common, serious condition that frequently results in morbidity and death and is a leading cause for hospital admissions. There is little evidence to guide ED physician disposition decisions for AHF patients. We sought to create a riskstratification tool for use by ED physicians to determine which AHF patients are at high risk for poor outcomes. Methods: We conducted a prospective cohort study in 9 tertiary hospital EDs and enrolled adult patients presenting with shortness of breath due to AHF. Patients were assessed for standardized clinical and laboratory variables and then followed to determine short-term serious outcome (SSO), defined as death, intubation, myocardial infarction, or relapse requiring admission within 14 days. We identified predictors of SSO by stepwise logistic regression and then rounded beta coefficients to create a risk scale. Results: We enrolled 1,733 patients with mean age 77.1 years, male $54.5 \%$, and initially admitted $50.1 \%$. SSOs occurred in $202(11.7 \%)$ cases $(14.0 \%$ in those admitted and $9.3 \%$ in those discharged from the ED). We created the CHFRS consisting of:1. Initial Assessment a) History of valvular heart disease b) On anti-arrhythmic c) Arrival heart rate $\geq 110 \mathrm{~d}$ ) Treated with non-invasive ventilation2. Investigations a) Urea $>12 \mathrm{mmol} / \mathrm{L}$ or $\mathrm{Cr}>150 \mu \mathrm{mol} / \mathrm{L}$ b) Serum $\mathrm{CO} 2>35$ $\mathrm{mmol} / \mathrm{L}$ or $\mathrm{pCO} 2>60 \mathrm{mmHg}$ (VBG or ABG) c) Troponin $>5 \mathrm{x}$ Upper Reference Level 3. Fails reassessment after ED treatment:(i) Resting vital signs abnormal, $(\mathrm{SaO} 2<90 \%$ on room air or usual O2, or HR $>110$, or RR $>28$ ); OR(ii) Unable to complete 3-minute walk test. The risk of SSO varied from $5.0 \%$ for a score of 0 , to $77.4 \%$ for a score of 9 . Discrimination between SSO and no SSO cases was good with an area under the ROC curve of 0.70 (95\% CI 0.66-0.74). There was good calibration between the observed and expected probability of SSO and internal validation showed the risk scores to be very accurate across 1,000 replications using the bootstrap method. Conclusion: We have created the CHFRS tool which consists of 8 simple variables and which estimates the short-term risk of SSOs in AHF patients. CHFRS should help improve and standardize admission practices, diminishing both unnecessary admissions for low-risk patients and unsafe discharge decisions for high-risk patients. This will ultimately lead to better safety for patients and more efficient use of hospital resources.

Keywords: heart failure, risk stratification, patient safety

\section{PL02}

Derivation and validation of a feasible emergency department specific frailty index to predict adverse outcomes

A. Brousseau, MD, E. Dent, PhD, R.E. Hubbard, MD, MSc, D. Melady, MD, M. Émond, MD, MSc, E. Mercier, MD, MSc, A. Costa, PhD,
Schwartz-Reisman Emergency Medicine Institute, Mount Sinai Hospital, Toronto, ON

Introduction: Frailty is an overarching concept in geriatric medicine. However its utility in the emergency department (ED) was not well understood. Objectives were to derive and validate an ED specific frailty index (FI-ED), using a cumulative deficits model; and to evaluate its ability to predict adverse outcomes. Methods: This was a large multinational prospective cohort study using data from: The Management of Older Persons in Emergency Departments (MOPED) and the interRAI study. The FI-ED was derived from the Canadian sample and validated in the multinational sample. Inclusion criteria were all patients $\geq 75$ years old presenting to an ED. The FI-ED used 24 variables identified in the interRAI ED-Contact Assessment tool, a brief focussed geriatric assessment. Its ability to predict adverse outcomes were analysed by logistic regression with odds ratio (OR). Results: There were 3903 participants: 2153 in the derivation sample and 1750 in the validation sample. In the derivation sample, increasing FI-ED was significantly associated with admission (OR 1.43 [95\% CI 1.34-1.52]), death in hospital (OR 1.55 [1.38-1.73]), prolonged hospital stay (OR 1.37 [1.22-1.54]), needs for Comprehensive Geriatric Assessment (OR 1.51 [1.41-1.60]) and discharge to long-term care (OR 1.30 [1.16-1.47]). In the validation sample, results were similar except for long-term care disposition (OR 0.84 [0.75 0.85]). Conclusion: The FI-ED conformed to characteristics previously reported in other geriatric populations. It was accurately derived and validated from a brief geriatric assessment feasible in the ED and can be used to predict adverse outcomes.

Keywords: frailty, geriatric, emergency

\section{PL03}

Implementation of the Canadian C-Spine Rule by paramedics: a safety evaluation

C. Vaillancourt, MD, MSc, M. Charette, MSc, J.E. Sinclair, MScN, J. Maloney, MD, R. Dionne, MD, P. Kelly, G.A. Wells, PhD, I.G. Stiell, MD, MSc, Ottawa Hospital Research Institute, Ottawa, ON

Introduction: The Canadian C-Spine Rule (CCR) was validated by emergency physicians and triage nurses to determine the need for radiography in alert and stable Emergency Department trauma patients. It was modified and validated for use by paramedics in 1,949 patients. The prehospital CCR calls for evaluation of active neck rotation if patients have none of 3 high-risk criteria and at least 1 of 4 low-risk criteria. This study evaluated the impact and safety of the implementation of the CCR by paramedics. Methods: This single-centre prospective cohort implementation study took place in Ottawa, Canada. Advanced and primary care paramedics received on-line and in-person training on the CCR, allowing them to use the CCR to evaluate eligible patients and selectively transport them without immobilization. We evaluated all consecutive eligible adult patients (GCS 15, stable vital signs) at risk for neck injury. Paramedics were required to complete a standardized study data form for each eligible patient evaluated. Study staff reviewed paramedic documentation and corresponding hospital records and diagnostic imaging reports. We followed all patients without initial radiologic evaluation for 30 days for referral to our spine service, or subsequent visit with radiologic evaluation. Analyses included sensitivity, specificity, kappa coefficient, t-test, and descriptive statistics with 95\% CIs. Results: The 4,034 patients enrolled between Jan. 2011 and Aug. 2015 were: mean age 43 (range 16-99), female 53.3\%, motor vehicle collision $51.9 \%$, fall $23.8 \%$, admitted to hospital $7.0 \%$, acute c-spine injury $0.8 \%$, and clinically important c-spine injury $(0.3 \%)$. The CCR classified patients for 11 important injuries with sensitivity $91 \%$ (95\% CI 58-100\%), and specificity 67\% (95\% CI 65-68\%). Kappa agreement for 
interpretation of the CCR between paramedics and study investigators was 0.94 (95\% CI 0.92-0.95). Paramedics were comfortable or very comfortable using the CCR in $89.8 \%$ of cases. Mean scene time was 3 min $(15.6 \%)$ shorter for those not immobilized ( $17 \mathrm{~min}$ vs. $20 \mathrm{~min}$; $\mathrm{p}=0.0001$ ). A total of $2,569(63.7 \%)$ immobilizations were safely avoided using the CCR. Conclusion: Paramedics could safely and accurately apply the CCR to low-risk trauma patients. This had a significant impact on scene times and the number of prehospital immobilizations.

Keywords: clinical decision rule, c-spine injury, emergency medical services

\section{PL04}

Initial serum lactate predicts deterioration in emergency department patients with sepsis

S.M. Fernando, MSc, D.P. Barnaby, MD, C.L. Herry, MD, A.J. Seely, MD, University of Ottawa, Department of Emergency Medicine, Ottawa, ON

Introduction: Early Emergency Department (ED) identification of septic patients at risk of subsequent deterioration is necessary in order to optimize disposition. High-risk patients admitted directly from the ED to the ICU have better outcomes than those admitted to the floor first. Initial ED serum lactate level has been associated with 28-day mortality in admitted patients, but there is little evidence on its use in predicting short-term deterioration. Furthermore, it is unclear whether the addition of respiratory rate (RR) to lactate would create a stronger predictive model of deterioration than either alone. Methods: Prospective cohort study of ED patients (age $\geq 18$ ) screened and treated for sepsis (defined as physician suspicion of infection and 2 or more of the SIRS criteria). Lactate and vital signs were obtained within 2 hours of ED arrival. Main outcome was deterioration (defined as any of the following: death; ICU admission $>24$ hours; Intubation; Vasoactive medications for $>1$ hour; or Non-invasive positive pressure ventilation for $>1$ hour) within 72 hours. Patients meeting an endpoint within 1 hour of arrival were excluded. Discharged patients were contacted at 72 hours to ensure that they had not met the endpoint or presented to another institution. Results: 985 patients presenting to either of two urban high-volume EDs were enrolled, of whom $84(8.5 \%)$ met the primary outcome. Initial serum lactate $\geq 4.0$ had a specificity of $97.4 \%$ (95\% CI, $94.1-100 \%$ ), but a sensitivity of $27.4 \%$ (95\% CI, 17-8-36.9\%) for predicting deterioration. Of patients with a lactate $\geq 4.0,4(8.7 \%)$ were discharged home, and did not reach an endpoint at 72 hours. Lactate $<2.0$ had a sensitivity of $95.5 \%$ (95\% CI: $93.4-97.1 \%$ ) and specificity of $84.5 \%$ (95\% CI: $80.4-88.6 \%$ ) for ruling out 72 -hour deterioration. Of patients with a lactate $<2.0,224(56.1 \%)$ were discharged home. Combining lactate with RR (AUC: 0.72, 95\% CI: 0.66-0.79) did not yield better predictive capability than lactate alone (AUC: $0.70,95 \%$ CI: $0.64-0.76$ ). Conclusion: Initial ED lactate is predictive of deterioration within 72 hours in patients with sepsis. The combination of lactate and RR was not more predictive of deterioration than lactate alone. This suggests that serum lactate has a role in predicting deterioration in patients with sepsis, and has utility in determining disposition.

Keywords: sepsis, risk stratification, lactate

\section{Oral Presentations}

\section{LO01}

Prevalence of pulmonary embolism among Canadian emergency department patients with syncope: a multicenter prospective cohort study

V. Thiruganasambandamoorthy, MD, MSc, M. Sivilotti, MSc, MD, A. McRae, MD, M.A. Mukarram, MBBS, MPH, S. Kim, BScH,
B.H. Rowe, MD, MSc, L. Huang, PhD, University of Ottawa, Department of Emergency Medicine, Ottawa, ON

Introduction: A recent cross-sectional study reported a $17.3 \%$ prevalence of pulmonary embolism (PE) among patients with syncope. However, the study had several flaws including spectrum and work-up bias with over-diagnosis due to excessive investigations. We sought to evaluate the prevalence of PE among Canadian emergency department (ED) patients presenting with syncope. Methods: We enrolled adults with syncope at 5 EDs and collected demographics, proportion of patients evaluated for suspected PE, their Wells PE score values and results of investigations [d-dimer, computed angiography (CT) of chest or ventilation-perfusion (VQ) scan]. 30-day adjudicated outcome included diagnosis of $\mathrm{PE}$ requiring treatment. We used descriptive statistics to report the results. Results: 4,739 patients [mean age 54.3 years, $54.4 \%$ females, and $587(12.4 \%)$ hospitalized] were enrolled. 323 patients $(6.8 \%)$ had further evaluation and investigations performed for suspected PE: 255 patients had D-dimer performed, 140 had CT chest and 17 had VQ performed. Of the 323 patients, 300 patients were low risk (Wells score $\leq 4$ ) and 23 were high-risk (score $>4$ ). A total of 16 patients $(0.3 \%)$ in the study cohort were diagnosed with PE: 10 patients were diagnosed in the ED, 5 patients were diagnosed while hospitalized as inpatient, and 1 patient was diagnosed on a return ED visit. Overall the prevalence of PE was $0.3 \%$ among all ED patients with syncope; and a $0.9 \%$ among those hospitalized for syncope. Conclusion: Our study shows that the prevalence of $\mathrm{PE}$ is very low among all patients presenting to the ED with syncope. The prevalence is also very low among those hospitalized for syncope than previously reported. While PE should be suspected and further investigations performed among syncope patients if clinically appropriate, caution should also be taken against indiscriminate overinvestigations for PE.

Keywords: syncope, pulmonary embolism, prevalence

\section{LO02}

Heart failure and palliative care in the emergency department M. Lipinski, MD, D. Eagles, MD, L.M. Fischer, MD, L. Mielneczuk, MD, I.G. Stiell, MD, MSc, University of Ottawa, Department of Emergency Medicine, Ottawa, ON

Introduction: Heart failure (HF) is a common ED presentation that is associated with significant morbidity and mortality. Despite recent evidence and recommendations for early palliative care (PC) involvement in these patients, they are still significantly under-served by PC services, often resulting in multiple ED visits. We sought to evaluate use of PC services in patients with HF presenting to the ED. Secondary objectives of the study were to investigate: 1) one year mortality, ED visits, and admissions; 2) application of a novel palliative care referral score. Methods: We conducted a health records review of 500 consecutive HF patients who presented to two academic hospital EDs. We included patients aged 65 years or older who were diagnosed as having a HF exacerbation by the emergency physician (ICD-10 code 150.-). Our primary outcome was PC involvement. Secondary outcomes included one year mortality rates, ED visits, admissions to hospital, as well as the application of a novel PC referral score developed by the institutional cardiac Palliative Care Committee. The score consisted of 6 different aspects of the patient's illness, including laboratory tests, hospital usage, and markers of decompensation. We conducted appropriate univariate analyses. Results: Patients were mean age 80.7 years, women $(53.2 \%)$, and had significant comorbidities (atrial fibrillation (51.2\%), diabetes $(40.4 \%)$ and COPD $(20.8 \%))$. Compared to those 